\title{
Preparation and characterization of novel chitosan-protamine nanoparticles for nucleus-targeted anticancer drug delivery
}

This article was published in the following Dove Press journal:

International Journal of Nanomedicine

14 November 2016

Number of times this article has been viewed

\author{
Xiwei Yu' \\ Jiahui Hou' \\ Yijie Shi' \\ Chang $\mathrm{Su}^{2}$ \\ Liang Zhao' \\ 'School of Pharmacy, ${ }^{2}$ School of \\ Veterinary Medicine, Jinzhou \\ Medical University, Jinzhou, People's \\ Republic of China
}

Correspondence: Liang Zhao School of Pharmacy, Jinzhou Medical Road, Linghe District, Jinzhou 121000 , People's Republic of China

Tel +864164673439

Fax +86 4164673439

Email liangzhao79@I63.com University, No.40, Section 3, Songpo

\begin{abstract}
It is well known that most anticancer drugs commonly show high toxicity to the DNA of tumor cells and exert effects by combining with the DNA or associated enzymes in the nucleus. Most developed drugs are first delivered into the cytoplasm and then transferred to the nucleus through the membrane pores. Sometimes, the transportation of drugs from cytoplasm to nucleus is not efficient and often results in poor therapeutic effects. In this study, we developed special and novel nanoparticles (NPs) made of chitosan and protamine for targeted nuclear capture of drugs to enhance anticancer effects. The anticancer effects of nuclear targeteddelivery of drugs in NPs were also evaluated by investigating cytotoxicity, cellular uptake mechanism, and cell apoptosis on cells. Chitosan-protamine NPs were characterized by good drug entrapment, sustained release, small average particle size, low polydispersity index, and high encapsulation efficiency; and accomplished the efficient nuclear delivery of fluorouracil (5-Fu). Compared with free 5-Fu and 5-Fu-loaded chitosan NPs, treatment of A549 cells and HeLa cells with 5-Fu-loaded chitosan-protamine NPs showed the highest cytotoxicity and further induced the significant apoptosis of cells. In addition, 5-Fu-loaded chitosan-protamine NPs exhibited the best efficiency in inhibiting tumor growth than the other three formulations. 5-Fu-loaded chitosan-protamine NPs enhanced antitumor efficacy through the targeted nuclear capture of drugs and showed promising potential as a nanodelivery system for quickly locating drugs in the nucleus of cells.
\end{abstract}

Keywords: nucleus, nanoparticles, chitosan, protamine, cytotoxicity

\section{Introduction}

It is well known that the main role for a therapeutic chemical drug during tumor treatment is to overcome a series of physiological barriers and be delivered into the specific intracellular situation and induce cell apoptosis. ${ }^{1-7}$ For some chemical nuclear-targeted drugs such as fluorouracil (5-Fu), principally used as a thymidylate synthase inhibitor for targeting the nucleus and interrupting the action of the enzyme for blocking synthesis of the pyrimidine thymidine and preventing DNA replication, ${ }^{8,9}$ various physiological barriers in the process must be overcome, in order to be transported into the nucleus to fulfill its antitumor effects. Therefore, identifying how to maximize the delivery and accumulation of drug in targeted nucleus is an important factor in improving its therapeutic effects. Generally, as 5-Fu can be transferred into cell nuclei through passive diffusion, the uptake of free 5 -Fu is time prolonged and limited by the decrease of drug concentration. Therefore, it is necessary to find a carrying vector to enhance its nuclear internalization and improve its curing effects. At present, biocompatible and biodegradable nanoparticles (NPs) as an effective drug delivery device is widely 
studied, and it is extensively used to deliver functional gene, protein, and drug compounds into cells. ${ }^{10-12}$

Drug-loaded NPs depend on enhanced permeability and retention effects to be easily aggregated around tumor sites. They are quickly transported into cells by internalization and enhance the concentration of drugs in tumor cells. ${ }^{13-15}$ In addition, compared to the rapid elimination of free drugs in vivo, drugs could be well protected by encapsulating in NPs, and their release could be regulated for longer retention in the body and thus achieve long-acting therapy. NPs in particular closely carry drugs and deliver the cargo from the cytoplasm into the nucleus through the nuclear membrane. ${ }^{16-20}$

Among candidates for a drug carrier system, chitosan as a kind of natural cationic polymer with its nontoxic, biocompatible, biodegradable, and antitumor properties is especially suitable to design a drug carrier for packaging some substances such as chemical compounds, vaccines, and genes. ${ }^{21-23}$ However, conventional chitosan NPs mainly deliver the drug to the cytoplasm. When some nucleus-targeted drugs are encapsulated in chitosan NPs and released into the cytoplasm, they are easily degraded by enzymes in cells and slowly transported from the cytoplasm into the nucleus by diffusion. Therefore, chitosan NPs need further modification to efficiently transport the nuclear worked drugs such as 5-Fu directly to the nucleus and improve the targeted delivery and therapeutic effects. It has been reported that protamines are small, arginine-rich, nuclear proteins containing a nuclear localization signal (NLS), which is an amino acid sequence consisting of one or more short sequences of positively charged lysines or arginines that could deliver proteins and chemical drugs to the cell nucleus by forming the nuclear pore complex, ${ }^{24-26}$ thus improving the uptake efficiency of exogenous substances into the cell nucleus. ${ }^{27,28}$

Based on protamine's precise nuclear localization, we developed novel NPs made of chitosan and protamine for targeted nuclear capture of drugs. The obtained chitosanprotamine NPs, having smaller particle size and positive charges, were specifically localized at cell nucleus as revealed by the intense endonuclear fluorescence. 5-Fu as a module drug was loaded into chitosan-protamine NPs for evaluating its antitumor effect. Compared to the lower cytotoxicity and apoptosis incurred by free 5-Fu and 5-Fu-loaded chitosan NPs, 5-Fu-loaded chitosan-protamine NPs increased cytotoxicity and induced significant apoptosis. This indicated that like some other nanomaterials, such as nanodiamonds, they were not able to completely penetrate the nucleus acting just on the cytoplasm. ${ }^{29-31}$ It is important to develop new
NPs of chitosan-protamine that can enter the nucleus and deliver the drug.

\section{Materials and methods Materials}

Chitosan with deacetylation degree of $80 \%$ and molecular weight of approximately $400 \mathrm{kDa}$, was purchased from Haixin Biological Product Co., Ltd (Ningbo, People's Republic of China). 5-Fu was purchased from Nantong Jinghua Pharmaceutical Co., Ltd (Nantong, People's Republic of China). Protamine with molecular weight of approximately $5.1 \mathrm{kDa}$ was purchased from Tianjin Sixth Pharmaceutical Co., Ltd (Tianjin, People's Republic of China); 3-(4,5-dimethylthiazol-2-yl)-2,5-diphenyltetrazolium bromide was obtained from Sigma-Aldrich (St Louis, MO, USA). All other chemicals purchased were of analytical grade and were obtained from a variety of vendors. A549 cells and HeLa cells were purchased from the Institute of Biochemistry and Cell Biology of Chinese Academy of Sciences (Shanghai, People's Republic of China).

\section{Preparation and determination of characteristics of chitosan-protamine NPs}

Accurately weighed $8 \mathrm{mg}$ chitosan was dissolved in $40 \mathrm{~mL}$ of $0.1 \%$ acetic acid solution followed by the addition of sodium hydroxide solution (20 wt \%) to adjust the $\mathrm{pH}$ of chitosan solution to 4.7. Then, $2 \mathrm{mg}$ protamine was dispersed in $4 \mathrm{~mL}$ ethanol under vigorous stirring, and chitosan solution and protamine solution were mixed together by magnetic stirring for 30 minutes followed by dropping addition of $1 \mathrm{mg} / \mathrm{mL}$ of sodium tripolyphosphate (TPP) until opalescence phenomenon appeared. It suggested that chitosan and protamine with protonated amino groups were conjugated with TPP, which have negatively charged group, to form NPs by electrostatic interactions. Removal of ethanol by vacuum triggered the self-precipitation of protamine in the aqueous phase and further compacted NPs to solid particles. To prepare drug-loaded NPs, $1 \mathrm{mg} 5$-Fu was pre-added into the mixture of chitosan and protamine before the dropping of TPP. The obtained NPs were centrifuged at $12,000 \mathrm{rpm}$ for 45 minutes in all cases and washed a further three times with distilled water to ensure complete removal of non-encapsulated drug. The pellets were finally resuspended in $1 \mathrm{~mL}$ of distilled water under ultrasonication and syringe-filtered through sterile nylon membrane filters of $0.20 \mu \mathrm{m}$ pore size. Particle size and polydispersity index (PDI) were evaluated by transmission electron microscope (JEM-1200EX; JEOL, Tokyo, Japan) 
and Zetasizer (Nano ZS90; Malvern, Malvern, UK). The encapsulation efficiency (EE) of the drug in NPs was carried out according to the protocol in our previous study, and in vitro drug release from NPs was estimated using a method reported previously. ${ }^{32}$

\section{Cell viability assays}

MTT cell proliferation assay kit provided a simple method for determination of cell number using standard microplate absorbance readers. Free 5-Fu, 5-Fu-loaded chitosan NPs, and 5-Fu-loaded chitosan-protamine NPs were chosen to incubate with cells for different periods at $37^{\circ} \mathrm{C}$ under $5 \%$ $\mathrm{CO}_{2}$. In all, $10 \mu \mathrm{L}$ of the $12 \mathrm{mM}$ MTT stock solution was added to each well and incubated for 6 hours at $37^{\circ} \mathrm{C}$ under $5 \% \mathrm{CO}_{2}$ and $95 \% \mathrm{O}_{2}$. Then, $50 \mu \mathrm{L}$ of dimethyl sulfoxide was added into each well and mixed thoroughly with the pipette, and incubated at $37^{\circ} \mathrm{C}$ for 10 minutes. The absorbance of the solution was quantified using a BioTek Synergy-2 microplate reader to measure absorbance at $490 \mathrm{~nm}$. Student's $t$-test was used for data statistics.

\section{Intracellular location of NPs in HeLa cells}

To analyze the intracellular localization (trajectories) of NPs, cells were grown in six-well chamber slides (BD Biosciences, Bedford, MA, USA). The nucleus was stained with Hoechst (blue) for 15 minutes at $37^{\circ} \mathrm{C}$, and fluorescein isothiocyanate (FITC) (green) was encapsulated in NPs for labeling. Different kinds of NPs entrapping FITC with the mass ratio of NPs and FITC at 50:1 were added into cells for incubation. The lysosome was stained by Lyso-Tracker Red DND-99. To reach the nucleus of the cells, NPs should show some mobility in the cytoplasm of the cells and their distributions were observed using confocal laser scanning microscopy (FluoView FV10i; Olympus, Tokyo, Japan).

\section{Endocytosis inhibition test}

To test which uptake mechanism was mainly dominated in the cellular entry of NPs, endocytosis inhibitors including chlorpromazine (inhibition of clathrin-mediated uptake) at $10 \mu \mathrm{g} / \mathrm{mL}$, genistein (caveolae-mediated uptake) at $1 \mu \mathrm{g} / \mathrm{mL}$, cytochalasin D (30 $\mu \mathrm{M}$, macropinocytosis), and $20 \mu \mathrm{g} / \mathrm{mL}$ sodium azide (an energy inhibitor) were preincubated with cells for 2 hours followed by the addition of FITC-labeled NPs with the mass ratio of NPs to FITC at 50:1 for continuous incubation. After 12 hours, extracellular NPs were removed followed by double washing with phosphate-buffered saline (PBS) and uptake ability of NPs was quantified using a microplate reader. The fluorescence from FITC was excited at $485 \mathrm{~nm}$ and emitted at $528 \mathrm{~nm}$. Endocytosis inhibition test was evaluated by calculating the uptake ratio of relative uptake rates of NPs treated with inhibitors and the uptake ratio of relative uptake rates of NPs treated with non-inhibitors. The relative uptake rates of NPs were determined by calculating the ratio of intracellular fluorescent intensity from internalized FITC-labeled NPs cells to the initial fluorescent intensity from the total added FITC-labeled NPs represented by relative fluorescent ratio (RFR, \%).

\section{Western blot assay}

In order to further evaluate the cell apoptosis effects induced by free drug and drug-loaded NPs, western blotting was performed to detect the expression levels of apoptosis-related proteins. The first step in a western blotting procedure is to separate the targeted proteins using gel electrophoresis. After electrophoresis, the separated targeted proteins are transferred or blotted onto a polyvinylidene difluoride membrane. Next, the membrane was blocked with bovine serum albumin to prevent any nonspecific binding of antibodies to the surface of the membrane. The membrane can then be further processed with primary antibodies specific for the target of interest and appropriate secondary antibody. After being stained with enhanced chemiluminescence, the levels of the targeted proteins were photographed and analyzed using a UVP gel analysis system (iBox Scientia 600; UVP, LLC, Upland, CA, USA).

\section{In vivo antitumor effect}

BALB/c nude mice (4-5 weeks, 13 \pm 1 g) were purchased from Beijing Vital River Laboratory Animal Technology Company, Beijing, People's Republic of China and randomly assigned to groups. All mice were inoculated subcutaneously with HeLa cells $\left(1 \times 10^{7}\right.$ cells suspended in $200 \mathrm{~mL}$ PBS $)$ to obtain the solid tumor. Twenty-four of the inoculated mice with the tumor volume of nearly $70 \mathrm{~mm}^{3}$ were evenly divided into four groups, which were treated with PBS, free 5-Fu, 5-Fu-loaded chitosan NPs, and 5-Fu-loaded chitosanprotamine NPs separately. Free drug and drug-loaded NPs with the same amount of 5-Fu at $1.6 \mathrm{mg}$ were re-suspended in $100 \mu \mathrm{L}$ PBS and injected subcutaneously into the anterior flank of mice for 5 consecutive days each week for 3 weeks. Three weeks later, all mice were sacrificed and tumor tissues were collected to evaluate antitumor efficacy. Tumor growth curves were determined by calculating the tumor volume using the formula $\mathrm{V}=\mathrm{a} \times \mathrm{b}^{2} \times \pi / 6$, where $\mathrm{a}$ is the length, $\mathrm{b}$ is the width, and $\mathrm{V}$ is given in $\mathrm{mm}^{3}$. The animal procedures performed followed the guidelines of National Institutes of 
Health Guide for the Care and Use of Laboratory Animals and were approved by the Animal Care and Use Committee of Jinzhou Medical University.

\section{Results}

\section{Preparation and determination of characteristics of 5-Fu-loaded chitosan-protamine NPs}

The obtained 5-Fu-loaded chitosan-protamine NPs prepared by ion gelation method were characterized of smaller particle size with the average size of $116 \pm 15 \mathrm{~nm}$, positive zeta potential about $27.8 \pm 4.21 \mathrm{mV}$, and lower polydispersity at $0.11 \pm 0.05$. In term of the EE of drugs, about $82.4 \% \pm 5.66 \%$ of 5-Fu were encapsulated in NPs (Figure 1 and Table 1). The results also showed that 5-Fu encapsulated in chitosanprotamine NPs was released slowly and smoothly in a biphasic drug release pattern at different $\mathrm{pHs}$, as shown in Figure 1C. Judging by the release curve of drug-loaded chitosan-protamine NPs, the drug release from NPs changed depending on the value of $\mathrm{pH}$, and when the value was lower, the release rate was faster. NPs also controlled the slow and smooth release of $5-\mathrm{Fu}$, and about more than $60 \%$ of total 5-Fu was slowly leaking out from NPs into medium within 48 hours at different $\mathrm{pH}$ levels. It suggested that in the acidic condition, the glucose amino group in chitosan chain bonded with $\mathrm{H}^{+}$to form $\mathrm{NH}^{3+}$ and dissolved in the medium rapidly, thus accelerating the breaking down of particle structure and resulting in fast diffusion of drug from the interior of NPs.

\section{MTT assay}

The cytotoxic effects of free 5-Fu and 5-Fu-loaded NPs were evaluated by MTT assay using A549 and HeLa cells (Figure 2). It was demonstrated that compared with free 5-Fu and 5-Fu-loaded chitosan NPs, 5-Fu-loaded chitosanprotamine NPs showed acute and significantly higher cytotoxicity in both the cells. In the initial incubation time from 6 hours to 12 hours, treatment of both the cells with free 5-Fu and 5-Fu-loaded chitosan NPs did not cause a significant decrease in the cell viability, indicating that free 5-Fu and 5-Fu-loaded chitosan NPs had been internalized into cells by simple penetration and endocytosis, and further formed endosomes that were fused with lysosomes to break down NPs activated by secreted lysosomal proteinases and released drugs. Therefore, it took a long time for free 5-Fu and 5-Fuloaded chitosan NPs to escape from lysosomes and were transferred into the cytoplasm. Thus, no obvious apoptotic effects were induced after treatment with free 5-Fu and 5-Fuloaded chitosan NPs over 12 hours at different concentrations of 5-Fu. With the extension of time, 5-Fu in the cytoplasm was gradually transported into the nucleus to promote significant apoptosis. The half-maximal inhibitory concentration $\left(\mathrm{IC}_{50}\right)$ values of 5-Fu-loaded chitosan NPs and free 5-Fu-treated A549 cells were $26.8 \mu \mathrm{g} / \mathrm{mL}$ and $29.6 \mu \mathrm{g} / \mathrm{mL}$ at 24 hours and $20.0 \mu \mathrm{g} / \mathrm{mL}$ and $22.3 \mu \mathrm{g} / \mathrm{mL}$ at 48 hours, respectively. The $\mathrm{IC}_{50}$ values of 5-Fu-loaded chitosan NPs and free 5-Fu-treated HeLa cells were $19.7 \mu \mathrm{g} / \mathrm{mL}$ and $21.8 \mu \mathrm{g} / \mathrm{mL}$ at 24 hours and $18.1 \mu \mathrm{g} / \mathrm{mL}$ and $19.4 \mu \mathrm{g} / \mathrm{mL}$ at 48 hours, respectively. Owing to the fact that protamine contains a NLS and has a
A

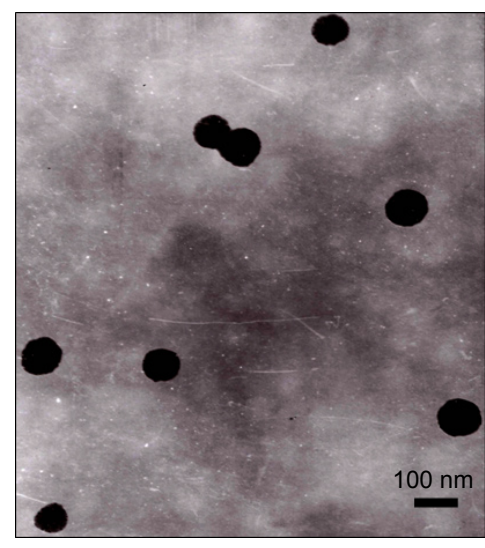

B

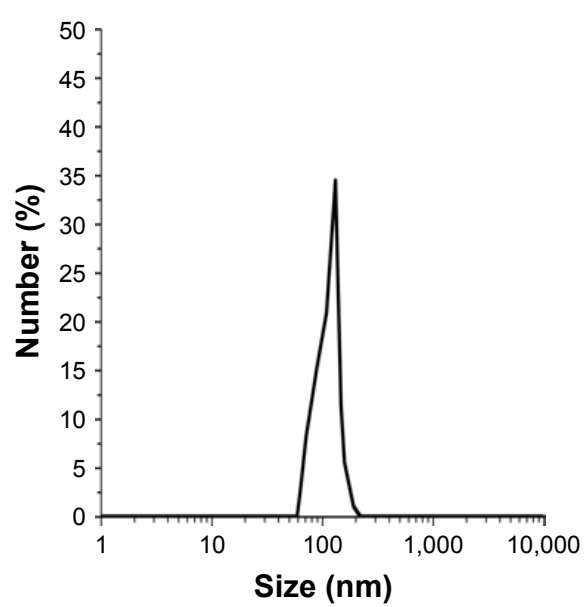

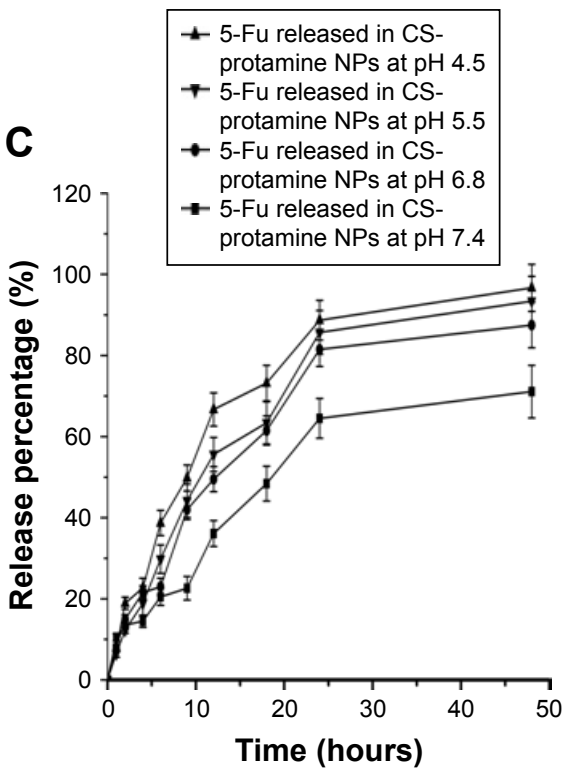

Figure I Characterization determination of 5-Fu-loaded chitosan-protamine NPs. TEM image of 5-Fu-loaded chitosan-protamine NPs (A), DLS analysis of the obtained 5-Fu-loaded chitosan-protamine NPs (B), and in vitro release profile of 5-Fu-loaded chitosan-protamine NPs in phosphate-buffered saline at $37^{\circ} \mathrm{C}$ for 48 hours (C). Abbreviations: CS, chitosan; DLS, dynamic light scattering; 5-Fu, fluorouracil; NP, nanoparticle; TEM, transmission electron microscope. 
Table I Key parameters of NPs

\begin{tabular}{|c|c|c|c|c|}
\hline Group & Diameter (nm) & Zeta potential $(\mathrm{mV})$ & Polydispersity & Encapsulation efficiency \% \\
\hline 5-Fu-loaded CS NPs & $100 \pm 9.3$ & $20.8 \pm 1.43$ & $0.24 \pm 0.08$ & $80.1 \pm 4.93$ \\
\hline 5-Fu-loaded CS-protamine NPs & $116 \pm 15$ & $27.8 \pm 4.21$ & $0.1 I \pm 0.05$ & $82.4 \pm 5.66$ \\
\hline
\end{tabular}

Note: Data presented as mean \pm SD.

Abbreviations: 5-Fu, fluorouracil; CS, chitosan; NP, nanoparticle.

promising potential to deliver cargo directly to the targeted nucleus, chitosan-protamine NPs could efficiently transfer 5-Fu into the nucleus in the shortest time, avoiding the degradation of lysosome and endocytosis by autophagosome. Therefore, 5-Fu-loaded chitosan-protamine NPs triggered rapid and severe cytotoxicity within a few hours of incubation at incubation concentrations. The $\mathrm{IC}_{50}$ values of $\mathrm{A} 549$ cells treated with 5-Fu-loaded chitosan-protamine NPs were $28.0 \mu \mathrm{g} / \mathrm{mL}$ at 6 hours, $26.5 \mu \mathrm{g} / \mathrm{mL}$ at 12 hours, $22.5 \mu \mathrm{g} / \mathrm{mL}$ at 24 hours, and $17.9 \mu \mathrm{g} / \mathrm{mL}$ at 48 hours. By contrast, the $\mathrm{IC}_{50}$ values of HeLa cells were $27.7 \mu \mathrm{g} / \mathrm{mL}$ at 6 hours, $22.5 \mu \mathrm{g} / \mathrm{mL}$ at 12 hours, $16.7 \mu \mathrm{g} / \mathrm{mL}$ at 24 hours, and $13.2 \mu \mathrm{g} / \mathrm{mL}$ at 48 hours. In order to justify that the blank chitosan-based NPs with positive potential had the cytotoxicity against A549 and HeLa cells, in vitro cytotoxicity of blank chitosan NPs and chitosan-protamine NPs had been studied. As shown in Figure 2, we found that inhibiting rates of blank chitosan NPs and chitosan-protamine NPs for tumor cells were very low and different amount of blank chitosan NPs, and chitosan-protamine NPs showed no obvious cell inhibition within 48 hours.

\section{Distribution of NPs in cells}

Based on the construction of carriers, the interactions between the carriers and targeted cells and their intracellular delivery process were further studied by using confocal laser scanning microscopy (FluoView FV10i; Olympus). FITC as a fluorescent marker was encapsulated in NPs to indicate the intracellular location of NPs and the nucleus was stained with Hoechst (blue) for 15 minutes at $37^{\circ} \mathrm{C}$, and the lysosome was stained with Lyso-Tracker Red DND-99. As shown in Figure 3, it was found that the green fluorescence of FITC was accumulated in cell surface after being treated with chitosan NPs or chitosan-protamine NPs for 3 hours, suggesting the initial efficient attachment of NPs with cell membrane in favor of further internalization. With the extension of incubation time, the green fluorescence from chitosan NPs was dispersed throughout the whole cytoplasm, and only a few weak green fluorescent dots were located in the nucleus. The stronger green color from chitosan-protamine NPs was mainly concentrated on the nucleus of HeLa cells. It demonstrated that compared to chitosan NPs, chitosan-protamine
NPs effectively promoted the nuclear translocation and constructed nanodelivery system for targeted drug transferring into cell nucleus. In the co-localization experiment, the result showed that red fluorescence representing lysosome was observed evenly inside the cells and the green color was accumulated on the cell surface, suggesting that there was no co-localization occurring between NPs and lysosome in the first 3 hours. With the extending incubation time at $37^{\circ} \mathrm{C}$, the increased green fluorescence denoting NPs was observed in the cytoplasm and colocated with the red fluorescence signifying lysosome. It was suggested that co-localization between chitosan NPs and lysosome was accelerated and the degree of lysosomal endocytosis of NPs was enhanced. It also indicated that chitosan NPs were mostly taken up by endocytosis and then entered lysosomal compartment. By contrast, when chitosan-protamine NPs were incubated with cells, small extent of co-localization between chitosanprotamine NPs and lysosome was observed. It could be explained that chitosan-protamine NPs could be efficiently transported into the cell nucleus in the shortest possible time and significantly reduced capture of chitosan-protamine NPs by lysosome; thus, chemical drugs can be delivered to the nucleus of the cells and prevent cargo from eventual lysosomal degradation.

\section{Uptake mechanisms involved in the cellular entry of NPs}

The internalization efficiency of NPs in cells was quantified by measuring RFR of the fluorescent intensity of internalized FITC-labeled NPs to that from the initially added FITClabeled NPs, expressed as a percentage. The results showed that when chitosan-protamine NPs were incubated with $\mathrm{HeLa}$ cells and A549 cells for 12 hours, the RFR observed in cells was increased gradually from less than $40 \%$ in the initial 3 hours to over $60 \%$ at 12 hours, as shown in Figure 4. It was suggested that chitosan-protamine NPs showed the similar intracellular uptake pattern in both cells and the internalization of NPs into the cell was time dependent. It is known that caveolae are ampullate (flask-shaped) invagination and abundance in the plasma membrane of many mammalian cell types and with the help of caveolin, carriers encapsulating drugs can be effectively transported to the interior of the cell, thus 


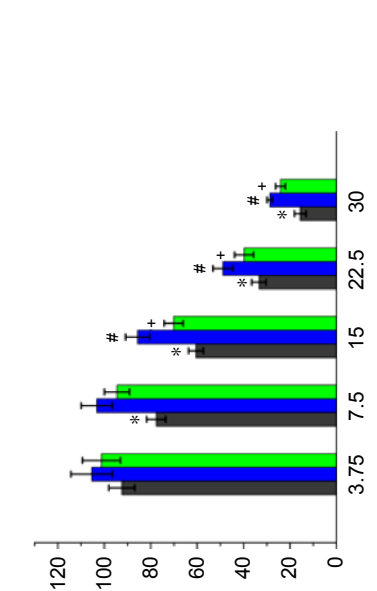

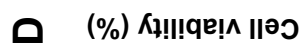

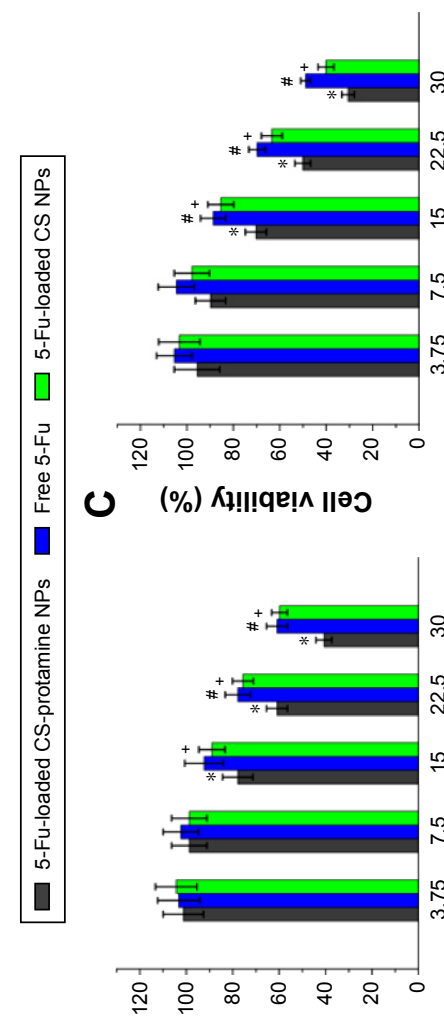

m (\%) Кใ!!!qए!̣ ॥әว

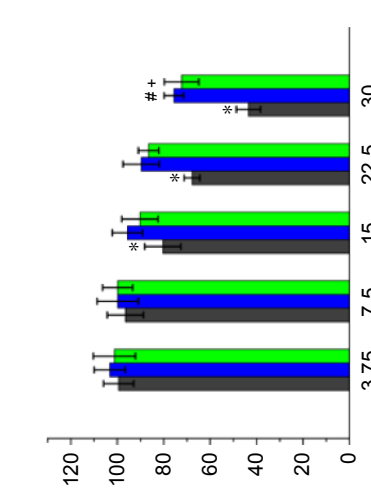

$\varangle \quad(\%)$ К ห!!!qu!^ ||әว

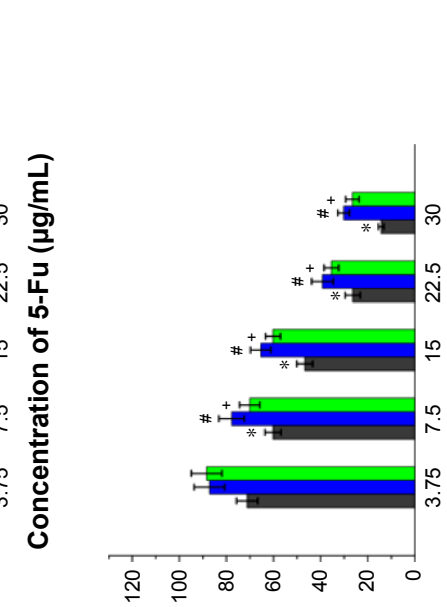

I (\%) К‼!qu!^ ||əว

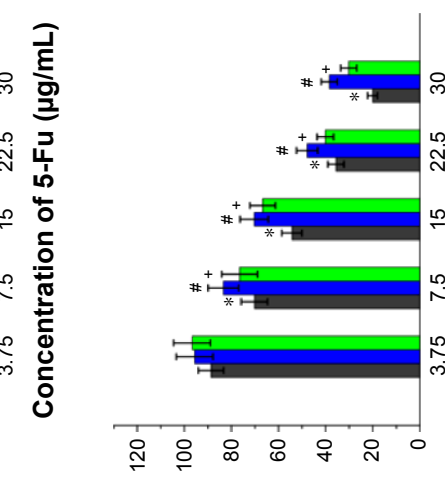

() (\%) К‼!q!! ॥ ॥əว

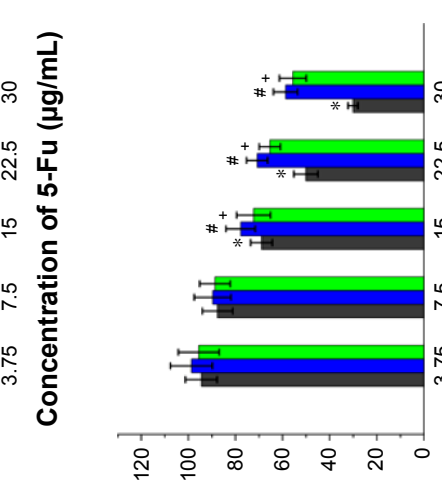

ᄂ (\%) К‼!qu!ฺ ||әว

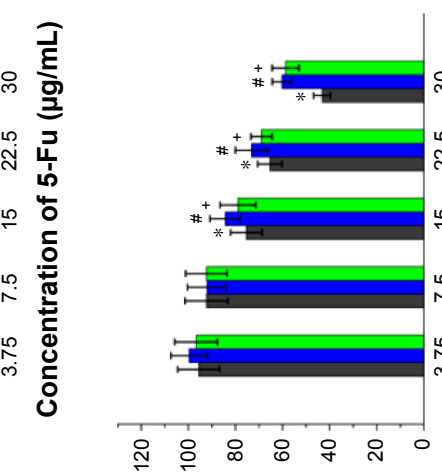

ш (\%) К‼!qu!^ ||әว

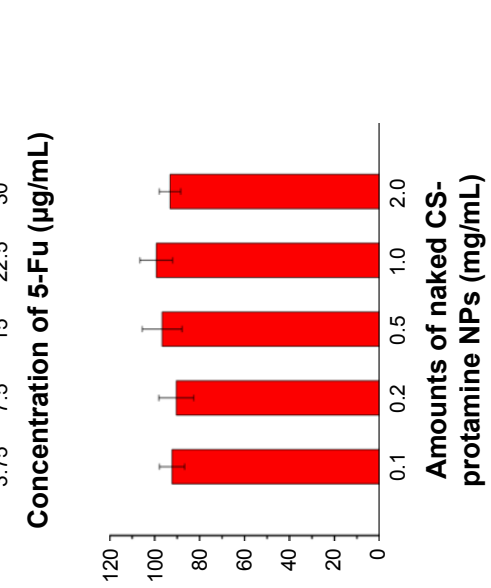

ـ

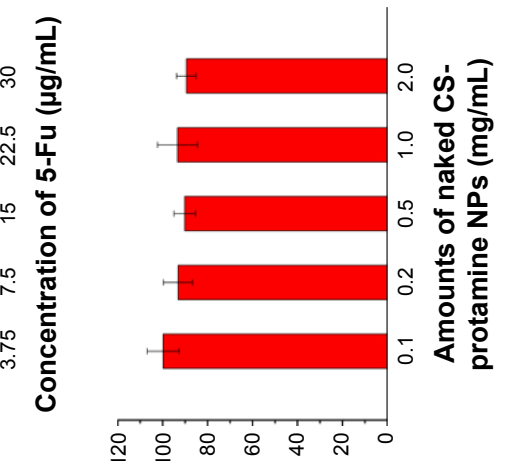

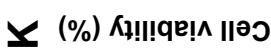

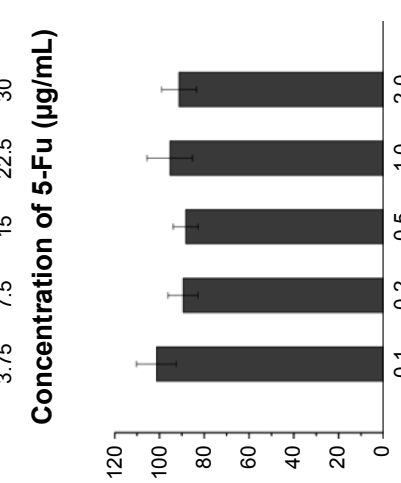

$\supset$ (\%) К‼!qस!^ ||әว

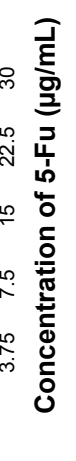

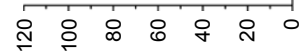

- (\%) Кใ!!!qu!^ ||әว

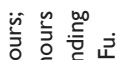

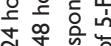

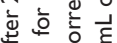

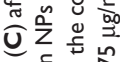

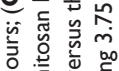

年

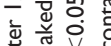

क.

空言㯊

은 텅흔

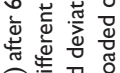

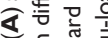

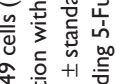

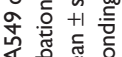

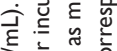

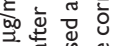

응

늘

ल)

음 퐁

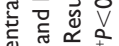

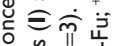

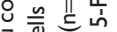

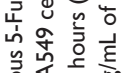

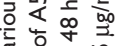

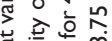

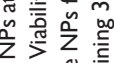

o

焉

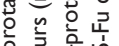

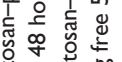

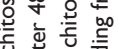

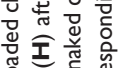

욱

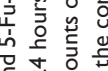

당

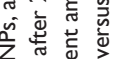

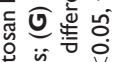

尊产产证

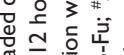

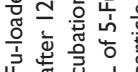

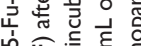

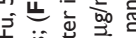

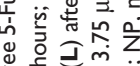

远

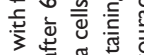

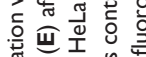

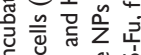

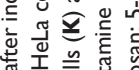

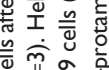

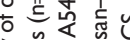

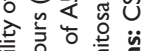

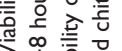

年

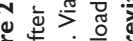

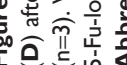



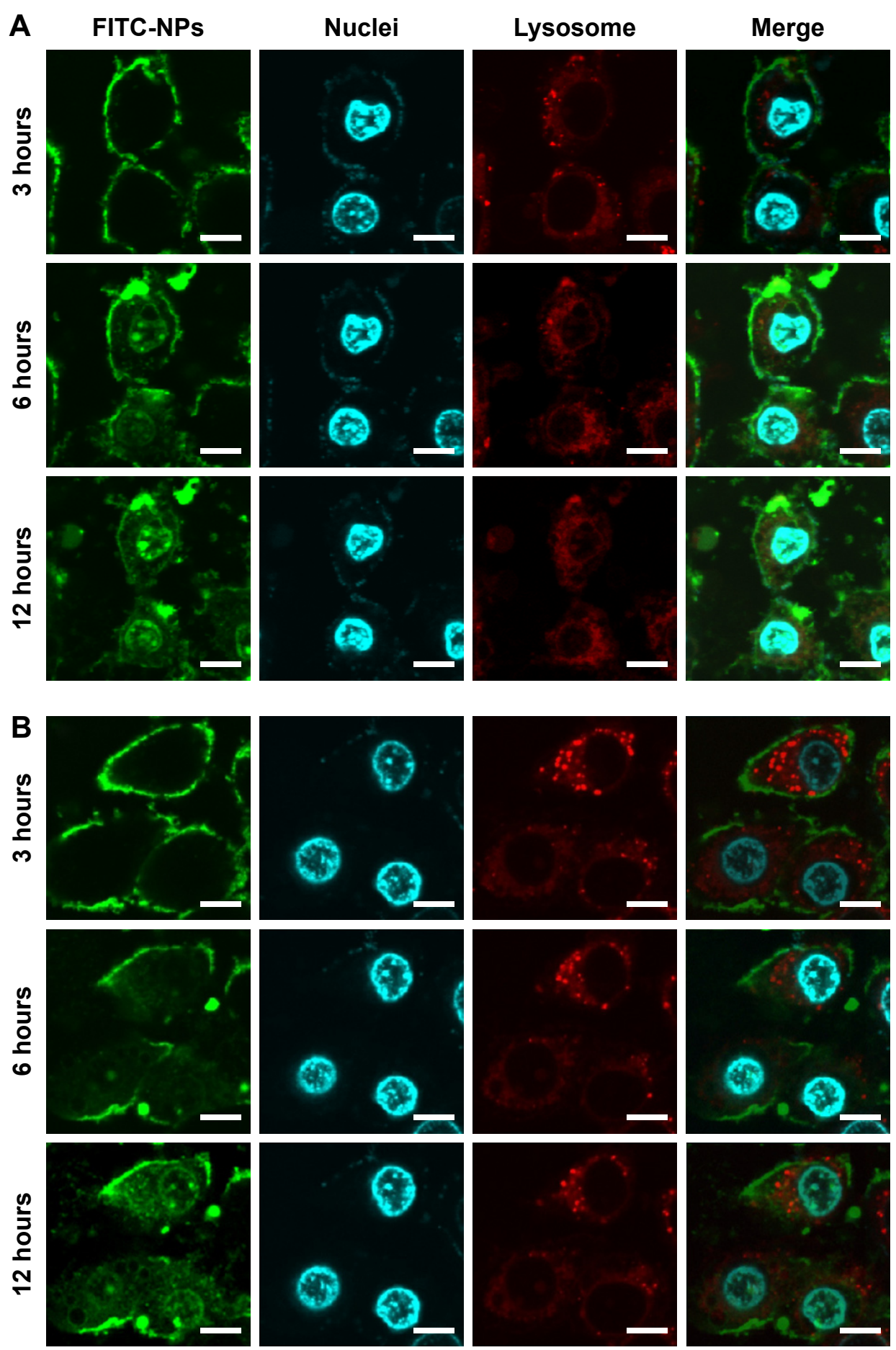

Figure 3 The uptake of FITC-labeled chitosan-protamine NPs (A) and FITC-labeled chitosan NPs (B) in HeLa cells after incubation for I2 hours with chitosan NPs and chitosan-protamine NPs. FITC as a green fluorescent marker was encapsulated in NPs to indicate the intracellular location of NPs; the nucleus was stained with Hoechst (blue) and the lysosome was stained with Lyso-Tracker Red DND-99 (red). The scale bar is $50 \mu \mathrm{m}$.

Abbreviations: FITC, fluorescein isothiocyanate; NP, nanoparticle.

avoiding degradation of drugs in the acidic organelles through bypassing the lysosome. Some inhibitors blocked different endocytic pathways and decreased the different level of cellular uptake of NPs and led to the difference in internalization efficacy. As shown in Figure 4, compared to that in untreated cells, the uptake of FITC-labeled NPs in A549 cells and HeLa cells pretreated with genistein for inhibiting caveolaemediated uptake were significantly reduced to $50.2 \%$ and $55.4 \%$ respectively, and the uptakes in cells after incubation with cytochalasin D for blocking macropinocytosis were 


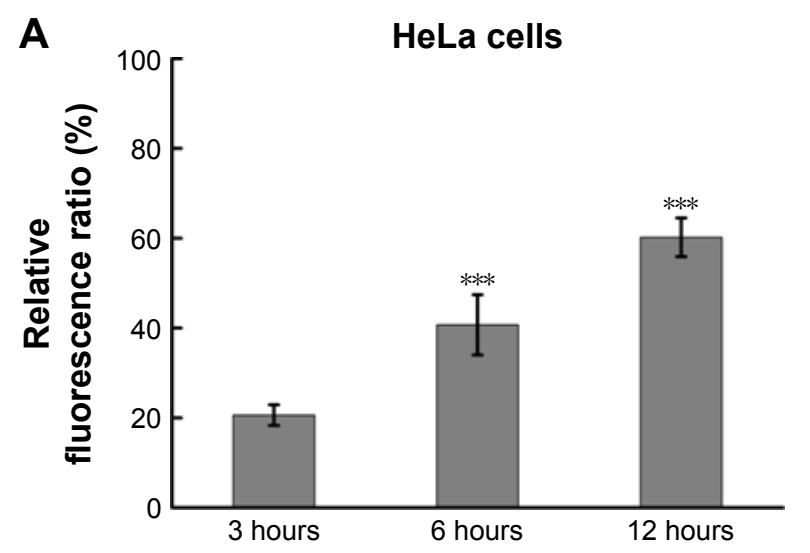

B

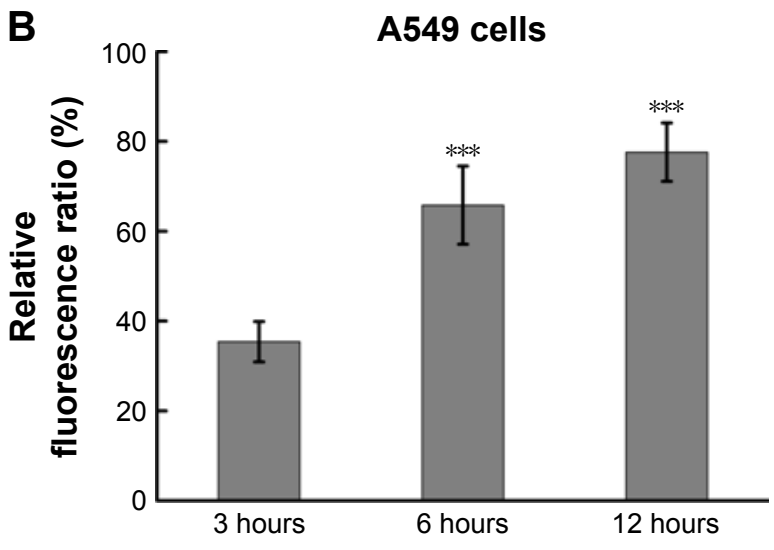

A549 cells

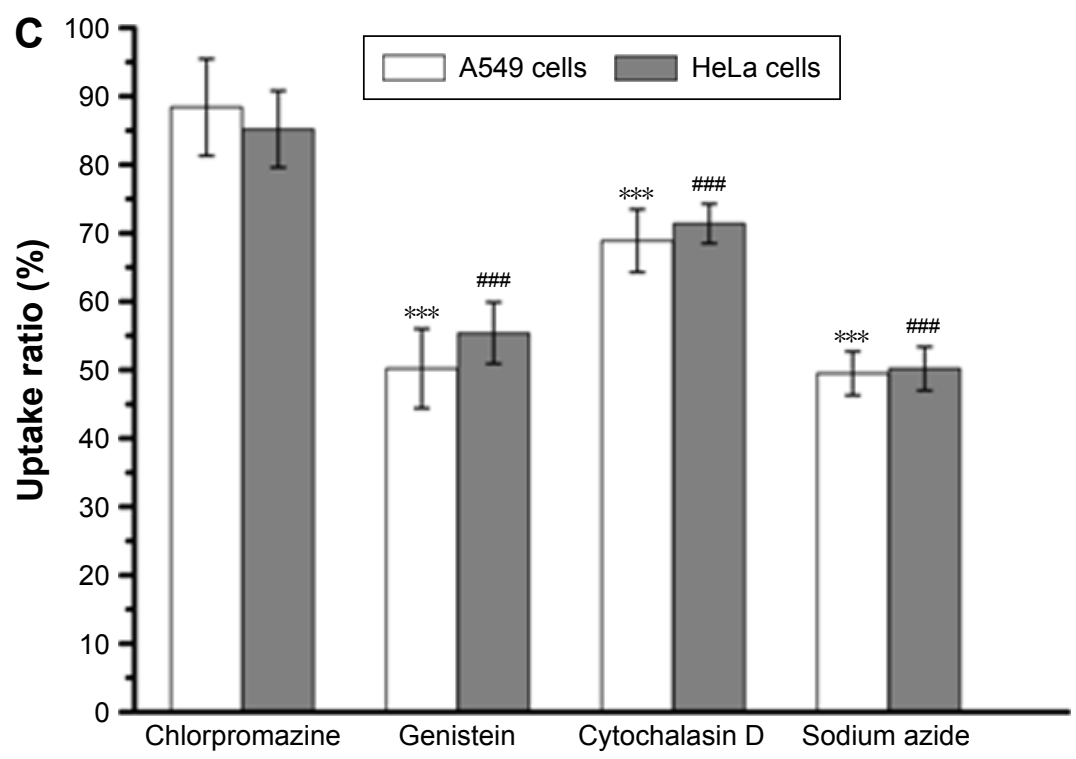

Figure 4 Fluorescence spectrum analysis of the uptake of chitosan-protamine NPs in HeLa cells (A) and A549 cells (B). Effects of endocytic inhibitors on the uptake ability of chitosan-protamine NPs in both cells (C). The endocytosis of chitosan-protamine NPs in cells was energy dependent and mainly relied on caveolae-mediated endocytosis and macropinocytosis uptake. Results are expressed as mean $\pm S D(n=3)$. ***P $<0.00$ I, vs FITC-labeled chitosan-protamine NPs treated with chlorpromazine in A549 cells. \# $<0.00$ I, vs FITC-labeled chitosan-protamine NPs treated with chlorpromazine in HeLa cells.

Abbreviations: NP, nanoparticle; SD, standard deviation; FITC, fluorescein isothiocyanate.

decreased to $68.9 \%$ and $71.4 \%$, respectively. Conversely, when pre-added into chlorpromazine for inhibiting clathrin-mediated endocytosis, no significant changes on uptake rates were found for chitosan-protamine NPs. The results showed that chitosan-protamine NPs were internalized through both caveolae-dependent endocytosis and macropinocytosis-dependent pathways. It was also supported by the observation that downregulation of intracellular ATP generation by sodium azide reduced the internalization of chitosan-protamine NPs considerably by about $49.5 \%$ in A549 cells and $50.2 \%$ in HeLa cells, whereas the endocytosis of NPs in cells was energy dependent.

\section{Western blot analysis}

In order to further evaluate apoptosis effects induced by free drug and drug-loaded NPs, western blot assay was adopted to determine the expression of apoptosis-related proteins such as Bax, cleaved caspase-3, and poly ADP-ribose polymerase (Parp). From Figure 5, it was found that 5-Fu-loaded NPs promoted higher apoptosis effects of both cells than free drugs represented by upregulated expression of Bax, Parp, and cleaved caspase-3 proteins. It suggested that the uptake of free 5-Fu depended on passive diffusion and was limited by the concentration gradient, in particular, intracellular 5-Fu without the protection of NPs was rapidly attacked by acidic lysosome, thus showing the lowest cytotoxicity. On the contrary, different from free 5-Fu, 5-Fu-loaded NPs were internalized through both caveolae-dependent endocytosis and macropinocytosis-dependent pathways and enhanced their cytotoxicity. Compared to the treatment group with the free 5-Fu and 5-Fu-loaded chitosan NPs, 5-Fu-loaded chitosan-protamine NPs induced the highest expression 
A
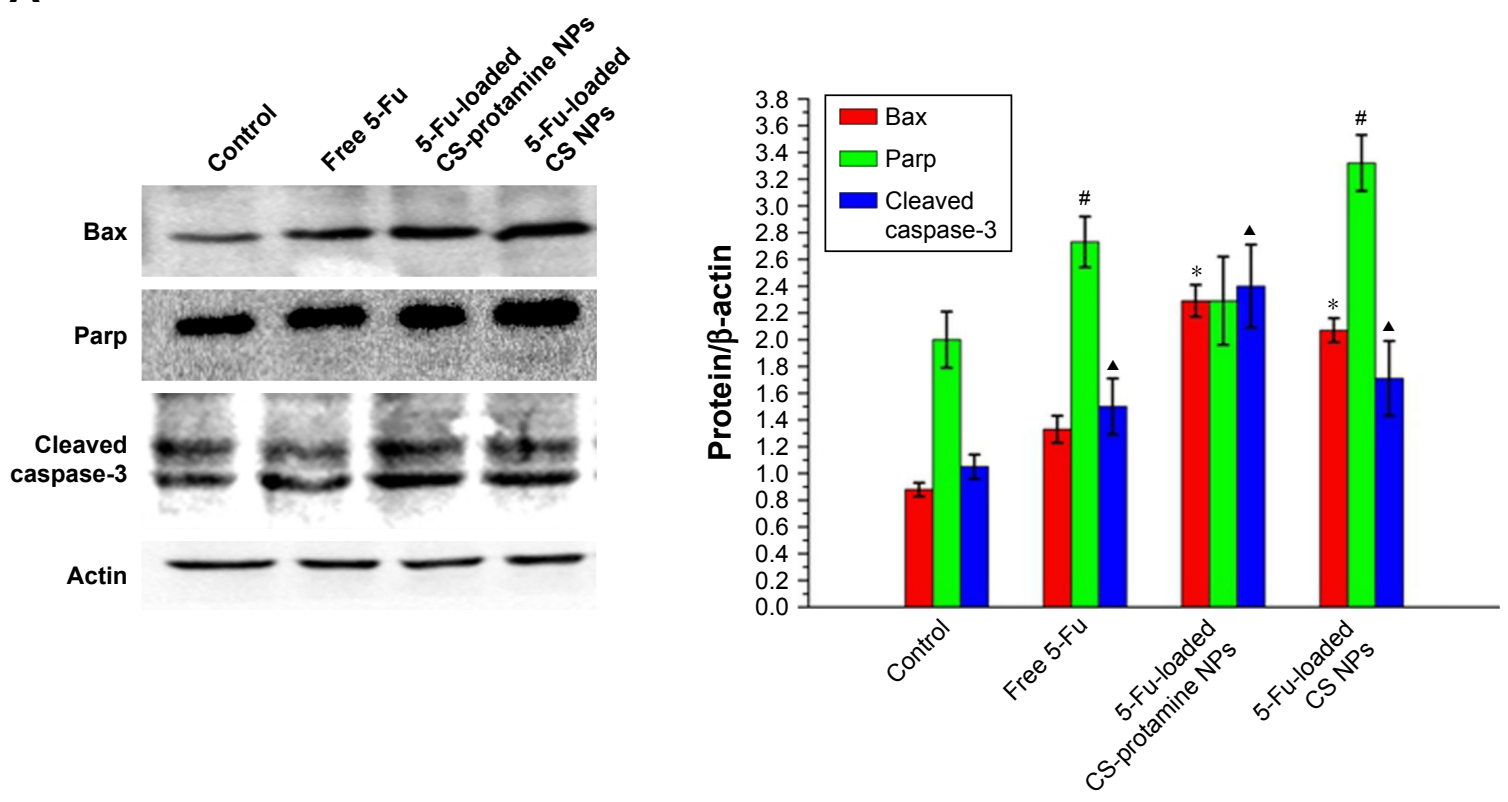

B
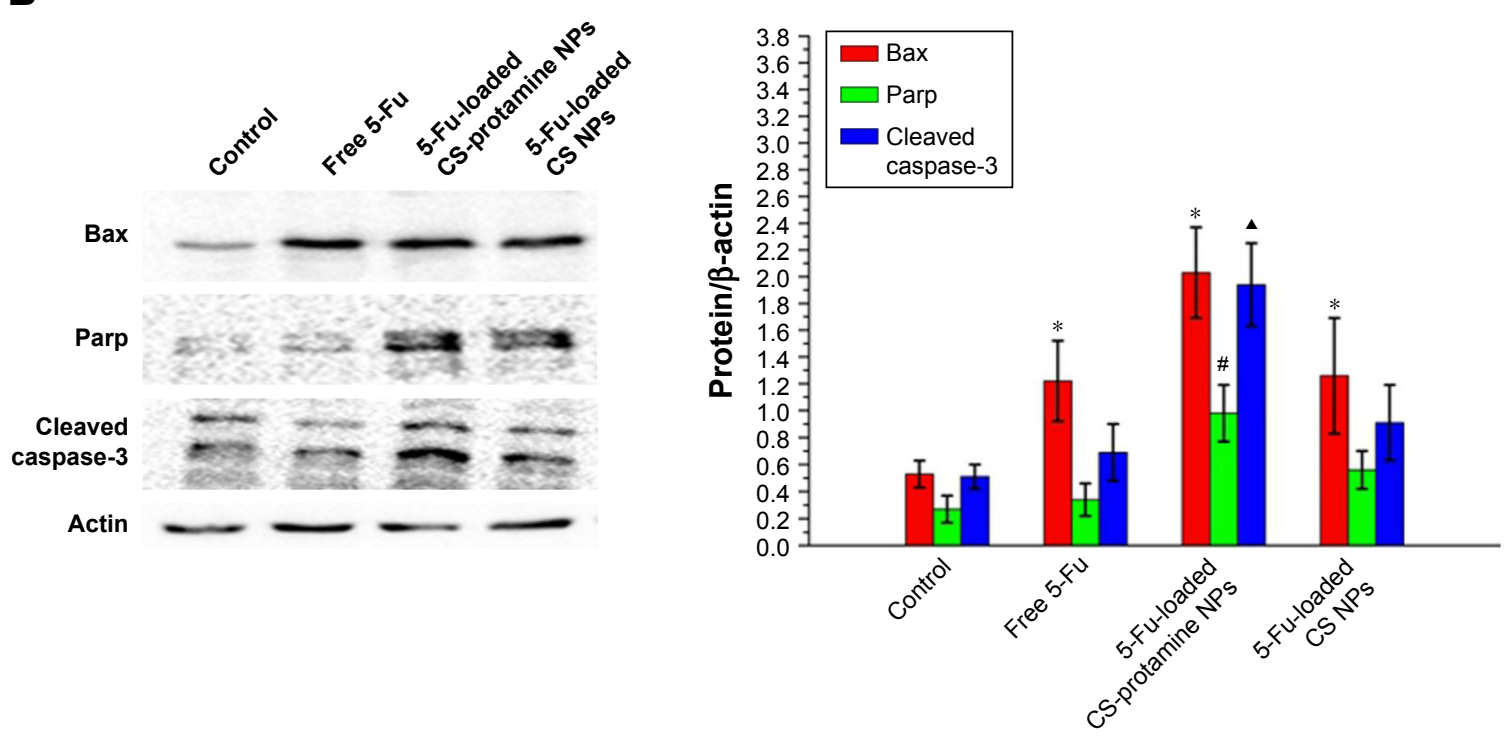

Figure 5 Apoptotic effects of various 5-Fu formulations on A549 cells (A) and HeLa cells (B). Western blot analyses of the expression levels of Bax, Parp, and cleaved caspase-3 proteins in cells after treatments. $* P<0.05$, vs the Bax protein expression of controlled group; ${ }^{\# P}<0.05$, vs the Parp expression of controlled group; $\mathbf{\Delta} P<0.05$, vs the cleaved caspase-3 protein expression of controlled group. Data are presented as mean $\pm S D(n=3)$.

Abbreviations: CS, chitosan; 5-Fu, fluorouracil; NP, nanoparticle; Parp, poly ADP-ribose polymerase; SD, standard deviation.

of cleaved caspase-3 in both cells. It proved that chitosanprotamine NPs significantly promoted targeted delivery of 5 -Fu into the nucleus and enhanced the concentration of 5 -Fu in cell nuclei, thus triggering the maximum apoptosis of cells.

\section{In vivo antitumor effect}

Antitumor efficacy of 5-Fu-loaded chitosan-protamine NPs in vivo in the mouse model bearing subcutaneous HeLa cell xenografts was evaluated by observing the volume change of tumor. It can be seen from Figure 6 that compared with the control group, after treatment with free 5-Fu and 5-Fuloaded chitosan NPs, the isolated tumor volumes were all smaller. More importantly, 5-Fu-loaded chitosan-protamine NPs caused a remarkable decrease in tumor volume, suggesting that 5-Fu-loaded chitosan-protamine NPs exhibited the best efficiency in inhibiting tumor growth than other three formulations. The result might be interpreted that more $5-\mathrm{Fu}$ had been released in the nucleus due to nucleus-internalization effect of chitosan-protamine NPs. 
A

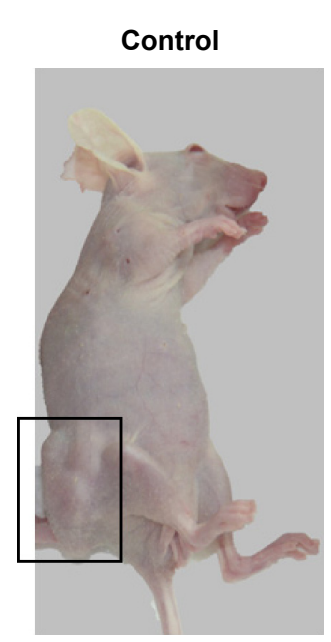

Free 5-Fu

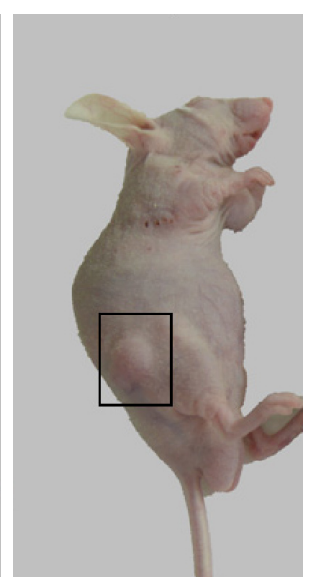

5-Fu-loaded CS NPs

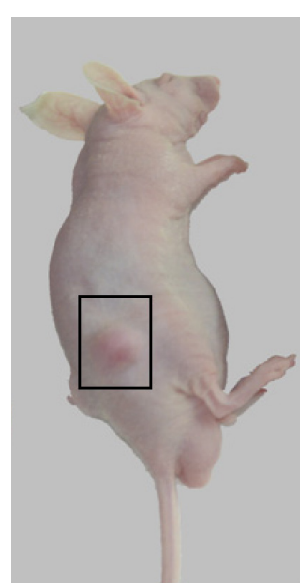

5-Fu-loaded

CS-protamine NPs

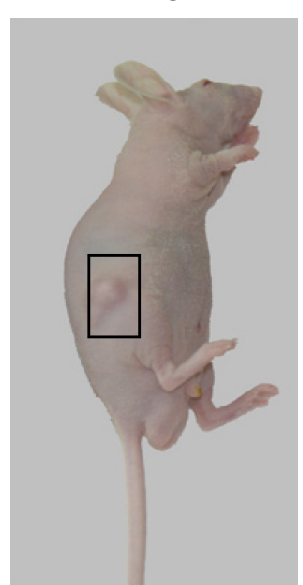

B

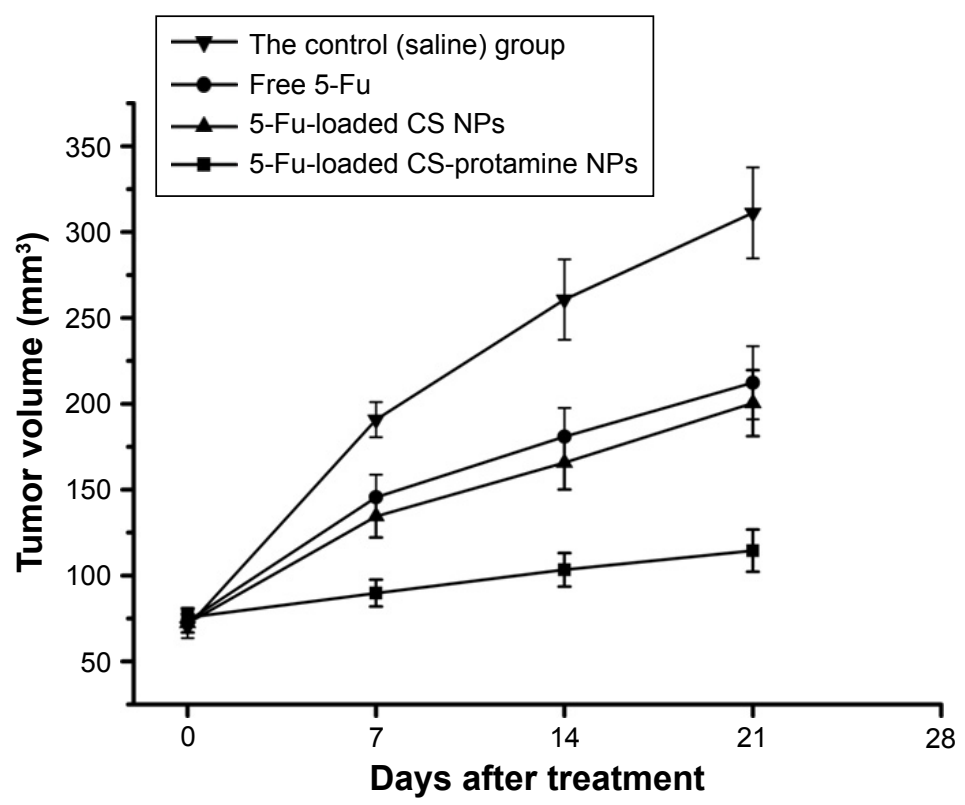

Figure 6 Images of mice bearing HeLa tumors on the 2 Ist day $(\mathbf{A})$ and tumor growth curves $(\mathbf{B})$. The rectangles indicate the HeLa cell tumors $(\mathbf{A})$, the tumor growth curves were determined by calculating the tumor volume using the formula $V=a \times b^{2} \times \pi / 6$, where $a$ is the length, $b$ is the width, and $V$ is given in $m m^{3}$.

Abbreviations: CS, chitosan; 5-Fu, fluorouracil; NP, nanoparticle.

Thereby, DNA synthesis was inhibited, cell apoptosis was accelerated, and tumor growth was suppressed. It further suggested that 5-Fu-loaded chitosan-protamine NPs as a potential drug delivery system enhanced the intracellular concentrations of drugs in the nucleus and improved the tumor suppression efficacy.

\section{Discussion}

Biodegradable NPs as a potential vector have acquired more attention due to low immune response and simplicity of preparation. They are easily synthesized and modified, and no immunogenicity and cytotoxicity are found. More importantly, they have overcome the various physiological barriers in the process of transportation of drugs and have achieved intracellular targeted localization to enhance the internalization efficiency and curing effects. We aimed to design special and novel NPs made of chitosan and protamine for targeted nuclear capture of drug to enhance anticancer effects. It has been proved in Figure 1 and Table 1 that protamine was blended with chitosan and formed matrix of NPs. Chitosan NPs and chitosan-protamine NPs had always a significant difference on intracellular distribution and physicochemical properties. Chitosan NPs began to diffuse into the cytoplasm and displayed enhanced green fluorescence. With the mediation of protamine as a NLS, chitosan-protamine NPs were mainly distributed in the 
cell nucleus. The zeta potential and particle size of chitosanprotamine NPs were about $27.8 \pm 4.21 \mathrm{mV}$ and $116 \pm 15 \mathrm{~nm}$, respectively, higher than that of chitosan NPs at about $20.8 \pm 1.43 \mathrm{mV}$ and $100 \pm 9.3 \mathrm{~nm}$, respectively. In fact, free 5-Fu could also be transferred into the nucleus through simple diffusion. 5-Fu encapsulated in chitosan NPs could be released into the cytoplasm from NPs by the degradation of chitosan NPs, and further diffused into the nucleus in the form of free 5-Fu. With the help of protamine, 5-Fu encapsulated in chitosan-protamine NPs could accomplish the efficient and active nuclear delivery by NLS peptidemediated NPs. To explore the apoptosis of 5-Fu-loaded NPs, western blot assay was performed to detect the expression of cleaved caspase-3. From Figure 5, it was found that compared to the treatment group with the free 5-Fu and 5-Fu-loaded chitosan NPs, 5-Fu-loaded chitosan-protamine NPs induced the highest expression of cleaved caspase-3 in HeLa cells and A549 cells. It was indicated that a large amount of drug-loaded chitosan-protamine NPs were accumulated in cells and specifically located in the nucleus due to the interaction between protamine and nuclei, leading to significant apoptosis and increasing expression level of cleaved caspase-3 proteins, as the main apoptosis-relevant protein, in western blot. It could also be confirmed by MTT results. Furthermore, 5-Fu-loaded chitosan-protamine NPs caused a remarkable decrease in tumor volume, suggesting that 5-Fu-loaded chitosan-protamine NPs exhibited the best efficiency in inhibiting tumor growth than other three formulations.

\section{Conclusion}

Chitosan-protamine NPs that we prepared were characterized by good drug entrapment, sustained release, smaller average particle size, low PDI, and high EE, and accomplished the efficient nuclear delivery of 5-Fu. Protamine was involved as a powerful tool for efficient nucleustargeted delivery of 5-Fu by NLS peptide-mediated NPs. Compared with free 5-Fu and 5-Fu-loaded chitosan NPs, treatment of A549 cells and HeLa cells with 5-Fu-loaded chitosan-protamine NPs showed the highest cytotoxicity and further induced significant apoptosis of cells. In addition, 5-Fu-loaded chitosan-protamine NPs exhibited the best efficiency in inhibiting tumor growth than other three formulations. Taken together, 5-Fu-loaded chitosanprotamine NPs enhanced antitumor efficacy through the targeted nuclear capture of drug and showed their promising potential as a nanodelivery system for locating drugs in the nucleus of cells.

\section{Acknowledgment}

This work was supported by Liao'ning Educational Committee (No L2014339) and Natural Science Foundation of Liaoning Province (No 2013022035).

\section{Disclosure}

The authors report no conflicts of interest in this work.

\section{References}

1. Cai X, Jin R, Wang J, et al. Bioreducible fluorinated peptide dendrimers capable of circumventing various physiological barriers for highly efficient and safe gene delivery. ACS Appl Mater Interfaces. 2016;8(9):5821-5832.

2. Pi Y, Zhou J, Wang J, et al. Strategies of overcoming the physiological barriers for tumor-targeted nano-sized drug delivery systems. Curr Pharm Des. 2015;21(42):6236-6245.

3. Chakrabarty P, Hudson Iii VJ, Sacino AN, et al. Inefficient induction and spread of seeded tau pathology in P301L mouse model of tauopathy suggests inherent physiological barriers to transmission. Acta Neuropathol. 2015;130(2):303-305.

4. Chakrabarti M, Ray SK. Anti-tumor activities of luteolin and silibinin in glioblastoma cells: overexpression of miR-7-1-3p augmented luteolin and silibinin to inhibit autophagy and induce apoptosis in glioblastoma in vivo. Apoptosis. 2016;21(3):312-328.

5. Lee BS, Cho YW, Kim GC, et al. Induced phenotype targeted therapy: radiation-induced apoptosis-targeted chemotherapy. J Natl Cancer Inst. 2014;107(2).pii:dju403.

6. Bao W, Liu R, Wang Y, et al. PLGA-PLL-PEG-Tf-based targeted nanoparticles drug delivery system enhance antitumor efficacy via intrinsic apoptosis pathway. Int J Nanomedicine. 2015;10:557-566.

7. Yuan Z, Syrkin G, Adem A, et al. Blockade of inhibitors of apoptosis (IAPs) in combination with tumor-targeted delivery of tumor necrosis factor- $\alpha$ leads to synergistic antitumor activity. Cancer Gene Ther. 2013;20(1):46-56.

8. Zhang N, Yin Y, Xu SJ, Chen WS. 5-Fluorouracil: mechanisms of resistance and reversal strategies. Molecules. 2008;13(8):1551-1569.

9. Matsusaka S, Lenz HJ. Pharmacogenomics of fluorouracil-based chemotherapy toxicity. Expert Opin Drug Metab Toxicol. 2015;11(5): 811-821.

10. Tanasienko IV, Yemets AI, Finiuk NS, Stoika RR, Blume YB. DMAEM-based cationic polymers as novel carriers for DNA delivery into cells. Cell Biol Int. 2015;39(3):243-245.

11. Jones $\mathrm{CH}$, Chen $\mathrm{CK}$, Chen $\mathrm{M}$, et al. PEGylated cationic polylactides for hybrid biosynthetic gene delivery. Mol Pharm. 2015;12(3):846-856.

12. Tangsangasaksri $M$, Takemoto $H$, Naito $M$, et al. siRNA-loaded polyion complex micelle decorated with charge-conversional polymer tuned to undergo stepwise response to intra-tumoral and intra-endosomal $\mathrm{pHs}$ for exerting enhanced RNAi efficacy. Biomacromolecules. 2016;17(1): 246-255.

13. Sun W, Fang N, Trewyn BG, et al. Endocytosis of a single mesoporous silica nanoparticle into a human lung cancer cell observed by differential interference contrast microscopy. Anal Bioanal Chem. 2008;391(6): $2119-2125$.

14. Deng X, Cao M, Zhang J, et al. Hyaluronic acid-chitosan nanoparticles for co-delivery of MiR-34a and doxorubicin in therapy against triple negative breast cancer. Biomaterials. 2014;35(14):4333-4344.

15. Du X, Xiong L, Dai S, Qiao SZ. $\gamma$-PGA-coated mesoporous silica nanoparticles with covalently attached prodrugs for enhanced cellular uptake and intracellular GSH-responsive release. Adv Healthc Mater. 2015;4(5):771-781.

16. Dam DH, Lee JH, Sisco PN, et al. Direct observation of nanoparticle-cancer cell nucleus interactions. ACS Nano. 2012;6(4): $3318-3326$. 
17. Tsuchiya A, Kanno T, Saito M, et al. Intracellularly transported adenosine induces apoptosis in [corrected] MCF-7 human breast cancer cells by accumulating AMID in the nucleus. Cancer Lett. 2012;321(1):65-72.

18. Zheng W, Yin T, Chen Q, et al. Co-delivery of Se nanoparticles and pooled SiRNAs for overcoming drug resistance mediated by P-glycoprotein and class III $\beta$-tubulin in drug-resistant breast cancers. Acta Biomater. 2016;31:197-210.

19. Song W, Popp L, Yang J, Kumar A, Gangoli VS, Segatori L. The autophagic response to polystyrene nanoparticles is mediated by transcription factor EB and depends on surface charge. J Nanobiotechnology. 2015;13:87

20. Duan J, Yu Y, Yu Y, et al. Silica nanoparticles induce autophagy and endothelial dysfunction via the PI3K/Akt/mTOR signaling pathway. Int J Nanomedicine. 2014;9:5131-5141.

21. Morris G, Kök S, Harding S, Adams G. Polysaccharide drug delivery systems based on pectin and chitosan. Biotechnol Genet Eng Rev. 2010;27:257-284

22. Jarmila V, Vavríková E. Chitosan derivatives with antimicrobial, antitumour and antioxidant activities - a review. Curr Pharm Des. 2011;17: 3596-3607.

23. Smith A, Perelman M, Hinchcliffe M. Chitosan: a promising safe and immune-enhancing adjuvant for intranasal vaccines. Hum Vaccin Immunother. 2014;10:797-807.

24. Kasinsky HE, Eirín-López JM, Ausió J. Protamines: structural complexity, evolution and chromatin patterning. Protein Pept Lett. 2011; 18(8):755-771.
25. Lewis JD, Saperas N, Song Y, Zamora MJ, Chiva M, Ausió J. Histone H1 and the origin of protamines. Proc Natl Acad Sci US A. 2004;101(12): 4148-4152.

26. Suzuki K, Ando T. Studies on protamines. XVI. The complete amino acid sequence of clupeine YII. J Biochem. 1972;72(6):1419-1432.

27. Tong HP, Wang LF, Guo YL, et al. Preparation of protamine cationic nanobubbles and experimental study of their physical properties and in vivo contrast enhancement. Ultrasound Med Biol. 2013;39(11): 2147-2157.

28. Samuel RE, Shukla A, Paik DH, et al. Osteoconductive protaminebased polyelectrolyte multilayer functionalized surfaces. Biomaterials. 2011;32(30):7491-7502.

29. Gismondi A, Nanni V, Reina G, Orlanducci S, Terranova ML, Canini1 A. Nanodiamonds coupled with 5,7-dimethoxycoumarin, a plant bioactive metabolite, interfere with the mitotic process in B16F10 cells altering the actin organization. Int J Nanomedicine. 2016;11:557-574.

30. Gismondi A, Reina G, Orlanducci S, et al. Nanodiamonds coupled with plant bioactive metabolites: a nanotech approach for cancer therapy. Biomaterials. 2015;38:22-35.

31. Gemeinhart RA, Luo D, Saltzman WM. Cellular fate of a modular DNA delivery system mediated by silica nanoparticles. Biotechnol Prog. 2005;21:532-537.

32. Zhao L, Su R, Cui W, Shi Y, Liu L, Su C. Preparation of biocompatible heat-labile enterotoxin subunit B-bovine serum albumin nanoparticles for improving tumor-targeted drug delivery via heat-labile enterotoxin subunit B mediation. Int J Nanomedicine. 2014;9:2149-2156.
International Journal of Nanomedicine

\section{Publish your work in this journal}

The International Journal of Nanomedicine is an international, peerreviewed journal focusing on the application of nanotechnology in diagnostics, therapeutics, and drug delivery systems throughout the biomedical field. This journal is indexed on PubMed Central, MedLine, CAS, SciSearch ${ }^{\circledR}$, Current Contents ${ }^{\circledR} /$ Clinical Medicine,

\section{Dovepress}

Journal Citation Reports/Science Edition, EMBase, Scopus and the Elsevier Bibliographic databases. The manuscript management system is completely online and includes a very quick and fair peer-review system, which is all easy to use. Visit http://www.dovepress.com/ testimonials.php to read real quotes from published authors. 